\title{
Prevalence, type, and correlates of trauma exposure among adolescent men and women in Soweto, South Africa: implications for HIV prevention
}

Kalysha Closson ${ }^{1,2}$, Janan Janine Dietrich ${ }^{3}$, Busi Nkala ${ }^{3,4}$, Addy Musuku', Zishan Cui ${ }^{2}$, Jason Chia ${ }^{2}$, Glenda Gray ${ }^{3}$, Nathan J. Lachowsky ${ }^{2,5}$, Robert S. Hogg ${ }^{1,2}$, Cari L. Miller ${ }^{1}$ and Angela Kaida ${ }^{1 *}$

\begin{abstract}
Background: Youth trauma exposure is associated with syndemic HIV risk. We measured lifetime prevalence, type, and correlates of trauma experience by gender among adolescents living in the HIV hyper-endemic setting of Soweto, South Africa.

Methods: Using data from the Botsha Bophelo Adolescent Health Survey (BBAHS), prevalence of "ever" experiencing a traumatic event among adolescents (aged 14-19) was assessed using a modified Traumatic Event Screening InventoryChild (TESI-C) scale (19 items, study alpha $=0.63$ ). We assessed self-reported number of potentially traumatic events (PTEs) experienced overall and by gender. Gender-stratified multivariable logistic regression models assessed independent correlates of 'high PTE score' ( $\geq 7$ PTEs).

Results: Overall, 767/830 (92\%) participants were included (58\% adolescent women). Nearly all (99.7\%) reported experiencing at least one PTE. Median PTE was 7 [Q1,Q3: 5-9], with no gender differences $(p=0.19)$. Adolescent men reported more violent PTEs (e.g." "seen an act of violence in the community") whereas women reported more nonviolent HIV/AIDS-related PTEs (e.g., "family member or someone close died of HIV/AIDS"). High PTE score was independently associated with high food insecurity among adolescent men and women $(\mathrm{aOR}=2.63,95 \% \mathrm{Cl}=1.36-5$. 09; $\mathrm{aOR}=2.57,95 \% \mathrm{Cl}=1.55-4.26$, respectively). For men, high PTE score was also associated with older age (aOR $=1$. 40/year, $95 \% \mathrm{Cl}=1.21-1.63)$; and recently moving to Soweto $(\mathrm{aOR}=2.78,95 \% \mathrm{Cl}=1.14-6.76)$. Among women, high PTE score was associated with depression using the CES-D scale $(\mathrm{aOR}=2.00,95 \% \mathrm{Cl}=1.31-3.03$,$) and inconsistent condom$ use vs. no sexual experience ( $\mathrm{aOR}=2.69,95 \% \mathrm{Cl}=1.66-4.37)$.

Conclusion: Nearly all adolescents in this study experienced trauma, with gendered differences in PTE types and correlates, but not prevalence. Exposure to PTEs were distributed along social and gendered axes. Among adolescent women, associations with depression and inconsistent condom use suggest pathways for HIV risk. HIV prevention interventions targeting adolescents must address the syndemics of trauma and HIV through the scale-up of gendertransformative, youth-centred, trauma-informed integrated HIV and mental health services.
\end{abstract}

Keywords: Adolescent, Young adult, Youth, HIV, Prevention, Trauma, Potentially traumatic events, Sexual and reproductive health, South Africa

\footnotetext{
* Correspondence: Kangela@sfu.ca

${ }^{1}$ Faculty of Health Sciences, Simon Fraser University (SFU), Blusson Hall Rm

10522, 8888 University Drive, Burnaby BC V5A 1S6, Canada

Full list of author information is available at the end of the article
}

(c) The Author(s). 2016 Open Access This article is distributed under the terms of the Creative Commons Attribution 4.0 International License (http://creativecommons.org/licenses/by/4.0/, which permits unrestricted use, distribution, and reproduction in any medium, provided you give appropriate credit to the original author(s) and the source, provide a link to the Creative Commons license, and indicate if changes were made. The Creative Commons Public Domain Dedication waiver (http://creativecommons.org/publicdomain/zero/1.0/) applies to the data made available in this article, unless otherwise stated. 


\section{Background}

South Africa has one of the highest rates of HIV globally, with an adult prevalence of $17.9 \%$ [1]. HIV disproportionately affects young people, and young women in particular. Among youth aged 15 to 24 years of age, $13.3 \%$ of young women and $3.8 \%$ of young men are living with HIV [2]. Addressing the high rate and burden of HIV among South African youth, and adolescent women in particular [3], is a national and global public health priority. While efforts are underway to scale-up access to several biomedical HIV prevention tools, including pre-exposure prophylaxis (PrEP), antiretroviral therapy (ART) for prevention ('TasP'), medical male circumcision, and female and male condoms [3, 4], demand for these programs will be shaped by the broader developmental, social and structural forces which influence adolescent sexual behaviour [5]. At present, there is a lack of literature on gendered differences in prevalence, types and influence of traumatic experiences and their relationship with adolescent HIV risk.

Experiences of childhood trauma are common among adolescents in South Africa, with estimates of physical and sexual violence in childhood ranging from 1.6$54.2 \%$ [6]. Traumatic experiences in childhood and adolescence have serious implications for short and long-term psychological and physical health outcomes, and have been associated with increased incidence of HIV [7-11]. The pathway from trauma and depression to heightened risk of HIV and other sexually transmitted infections has been described through the negative effects of depression on impulse control, risk perception [12], self-esteem and self-efficacy [13], substance use [14], and socio-structural vulnerability [15], which compromise HIV prevention behaviours $[16,17]$. Such pathways are highly gendered, with both the prevalence of depression and associations with increased risk of condomless sex shown to be higher among adolescent women than adolescent men [18].

The disproportionate exposure to potentially traumatic events (PTEs) experienced by people living with HIV (PLHIV), has been referred to as a syndemic ("synergistically interacting epidemics") [19], yielding a range of poor social, clinical, and public health outcomes, including decreased social functioning, elevated rates of posttraumatic stress disorder (PTSD), increased prevalence of high-risk sexual and drug use behaviours, suboptimal adherence to ART, poor HIV clinical outcomes, increased HIV transmission risk, and higher mortality $[7,9,10]$. Little attention, however, has focused on gendered impacts and the presence of syndemic risks which can have a multiplicative effect on HIV risk [20], including multiple types of PTEs (e.g. physical, sexual, and emotional) [21].

Adolescent men and women are exposed to different types and consequences of trauma, particularly with respect to violent and non-violent forms. Globally, violence against women is a major social justice issue [22, 23], an under-addressed public health priority, and an established risk factor for HIV acquisition and other negative health outcomes [3, 24]. In South Africa, where reports of violence are known to underestimate the true prevalence [25], 20\% of women attending antenatal care reported experiencing sexual violence, among the highest prevalence in the world $[22,26]$. Among adolescent men, experiences of perpetrating or witnessing interpersonal violence drive rates of trauma exposure [11, 24, 27]. This is significant as earlier research among South African adolescent men demonstrated an association between witnessing community violence and high sexual HIV risk behaviours such as multiple concurrent sexual partnerships [28].

The effects of experiencing trauma on mental health and coping strategies also differ between adolescent men and women in ways that influence HIV risk pathways. For instance, PTEs experienced by South African women have been shown to increase internalized behaviours such as depression, anxiety and PTSD [23, 29], which synergistically contribute to increased risk for HIV and other sexually transmitted infections (STIs) $[26,30]$. However, adolescent men are more likely to respond to PTEs with adverse externalized behaviours that introduce HIV risk, including delinquency, aggression and substance abuse [21]. This distinction in type of PTEs and behavioural responses demands gender-specific analysis, support, and response.

We measured the lifetime prevalence and correlates of PTEs overall, and by gender among adolescent men and women in Soweto, South Africa. This information is critical to inform youth-centred sexual and reproductive health and HIV prevention programming that considers the broader risk environments that youth navigate [31].

\section{Methods \\ Study setting}

We used cross-sectional survey data from adolescents (aged 14-19 years) enrolled in the Botsha Bophelo Adolescent Health Study (BBAHS) in Soweto, South Africa. Soweto is a large township southwest of Johannesburg with a population of approximately 1.3 million predominantly (98.5\%) black inhabitants residing in informal and formal settlements [32]. While there are no populationlevel statistics on HIV prevalence among adolescents in Soweto, a recent study of 11,552 adolescents and young adults (14-25 years) residing in Soweto, reported that 4\% of those who accessed HIV testing services at a local youth-centered clinic tested positive for HIV, including $2 \%$ of young men $4 \%$ of young women [33]. 
BBAHS was conducted at the Perinatal Health Research Unit (PHRU) and the Kganya Motsha Adolescent Centre (KMAC) in Soweto, South Africa. KMAC was opened in 2008 with a local mandate to address HIV and sexual and reproductive health priorities of adolescents (ages 14-19 years). Earlier pilot studies on adolescent health identified the urgent need for such youth-centred services, and informed the development and implementation of BBAHS [33-36].

\section{Study participants}

Adolescents aged 14-19 years residing in Soweto were eligible to participate in BBAHS. Participants were recruited from across 41 townships to be representative of adolescents living in formal and informal communities within Soweto. Participant recruitment occurred around local malls, schools, neighbourhood hangouts, through peer-word-of-mouth, and staff outreach. We used a targeted stratified sampling and recruitment approach, based on geographic location, age, and gender. In order to reflect the gendered dimensions of HIV risk in South Africa, we aimed for a sample comprised of $60 \%$ young women and $40 \%$ young men. The research team approached interested adolescents for participation, and if eligible, were enrolled in the study. A total of 956 interviews were completed between March 2010 and March 2012. This amount of recruitment time was required to meet stratified sampling targets, and to ensure inclusion of youth from more remotely located townships with Soweto and harder-to-reach youth subpopulations. Of 956 completed interviews, $\mathrm{n}=126$ were excluded as they were determined to be outside of the targeted age criteria or had incomplete data, yielding a final sample of 830 adolescent participants. Additional information about the study procedures of the BBAHS can be found elsewhere [37].

\section{Ethical considerations}

Adolescents under 18 years signed an informed assent form and provided a signed informed consent form from a parent or legal guardian. Adolescents aged 18 or 19 signed an informed consent form. Age was verified using birth certificates or other identity documents.

Ethical approval for the study was granted by the ethics committees of the University of the Witwatersrand (Johannesburg, South Africa) and Simon Fraser University (Burnaby, Canada).

\section{Data collection}

An interviewer-administered, structured, online questionnaire was delivered to participants (supported by SurveyMonkey ${ }^{\mathrm{TM}}$ software) via iPad or desktop computer. Interviewers received extensive training in good clinical practice guidelines, participant recruitment, administering questionnaires, and participant referral in cases where additional support was required after the study visit. Interviews were conducted in either English or isiZulu at the PHRU, the KMAC, or at a private location selected by the participant. Questionnaires took an average of $60 \mathrm{~min}$ to complete, and participants received 50 Rand (approximately 7 USD at the time) as compensation for their time and transportation costs. An international team of experts in adolescent health and HIV, including an adolescent Community Advisory Board $(\mathrm{CAB})$, contributed to the development of the BBAHS questionnaire [37].

\section{Measures}

\section{Primary outcome: trauma experience}

Assessment of 'trauma experience' followed Norris' [29] comprehensive definition of traumatic events as "any event that produces symptoms of traumatic stress" (23, p. 409). We measured PTEs using a modified version of the Traumatic Events Screening Inventory-Child (TESIC) [29]. Unlike other trauma scales, the TESI-C scale was developed to be language appropriate for children and youth.

The TESI-C measures the history of trauma by asking about exposure ("yes" vs. "no") to twenty PTEs including "injuries, hospitalizations, domestic violence, community violence, disasters, accidents, physical abuse and sexual abuse" [38]. Historically, this scale has been used in child and adolescent psychological screening [38]. For our study, the TESI-C items were modified to account for the social context and physical environment of adolescents in Soweto [38]. For example, TESI-C items regarding natural disasters, acts of war or terrorism, kidnapping and animal attacks were omitted. Similar to other South African studies examining the impact of traumatic experiences in adolescents, we added items regarding parents separating, parents arguing, changing schools, parents' job security, family members with HIV/AIDS, family members dying of HIV/AIDS, discrimination, financial security, personal physical attack were added. The final adapted scale included a total of 19 items (study alpha $=0.63$; Table 2 ). A comparison of items from the original TESI-C scale and the modified version used in this analysis is included in the Additional file 1.

We measured prevalence of experiencing a potentially traumatic event (i.e., a response of "Yes" to one or more of the 19 items included in the modified TESI-C scale) overall and by gender. We also assessed number of reported PTEs and calculated a PTE score (range $=0-19$ ), with higher scores indicating higher PTE experience. Scores greater than the scale median were considered 'high PTE score' vs. 'low PTE score'. 


\section{Explanatory factors}

Socio-demographic characteristics We assessed sociodemographic characteristics by gender (adolescent man vs. adolescent woman), age in years (continuous), ethnicity (Zulu, Xhosa, Sotho, Tswana or other), education (high school or greater vs. less than high school), and employment (student vs. unemployed vs. employed [fulltime/part-time/self-employed]). Additional determinants of socio-economic status included length of time living in Soweto ( $<5$ years vs. $\geq 5$ years vs. since birth), housing type (brick house or flat owned by family vs. brick house or flat rented by family or other housing type vs. reconstructive development housing [RDP] or shack), food insecurity (low vs. medium vs. high, measured via a 9-item hunger and food security scale [39] [study Cronbach's $\alpha$ $=0.81]$ ), and receiving a household social grant in the past 12-months (yes vs. no; including disability, age pension, child support or other social grant), and history of incarceration (ever vs. never).

Depression The 20-point Center for Epidemiologic Studies Depression $(C E S-D)$ Scale was utilized to measure probable depression (study Cronbach's $\alpha=0.81$, range $=0-60$, with higher scores indicating greater depressive symptoms) [40]. In the general population the American Psychological Association suggests using a cut off of 16 or higher to determine major depressive disorder [41]. We chose a higher cut off of $\geq 24$ as this has been previously described as the best cut-off to determined 'probable depression' among adolescents [18, 42].

Sexual behaviour History of sexual activity was defined by participant report of ever having had intercourse (yes vs. no), current sexual activity was defined as having had sex (vaginal or anal) in the 6 months prior to interview (yes vs. no) and, if yes, whether the participant had more than one sexual partner in the last 6 months (yes vs. no). Consistent condom use was assessed via self-reported lifetime use during anal and/or vaginal sex, as applicable, and frequency (always vs.vs sometimes vs. never) in the 6 months prior to interview (lifetime consistent condom use vs. any inconsistent or no condom use vs. never had sex). History of STI diagnosis and/or symptoms (ever vs. never), history of HIV testing (ever vs. never), and HIV status (HIV-positive vs. HIV-negative vs. unknown HIV status) was assessed via self-report.

Substance use We assessed self-reported frequency of alcohol use in the 6 months prior to interview (once a month or more vs. less than once a month or never). We also assessed any use of illicit (e.g., heroin, cocaine, ecstasy) or licit drugs used in a manner other than which they are prescribed (e.g., prescription pills, antiretrovials/ whoonga), excluding marijuana in the 6 months prior to interview (yes vs. no). Use of marijuana (yes vs. no) was assessed separately, given different patterns of use among youth [43, 44].

\section{Statistical analysis}

All analyses were conducted using SAS 9.4, stratified by self-identified gender. Descriptive statistics (median, $1^{\text {st }}$ quartile [Q1] and $3^{\text {rd }}$ quartile [Q3] for continuous variables and $\mathrm{n}, \%$ for categorical variables) were used to characterize baseline distributions of study variables. Differences in baseline variables and trauma scores by gender were compared using Wilcoxon rank sum test for continuous variables and Pearson $\chi^{2}$ or Fisher's exact test for categorical variables.

Univariable and multivariable logistic regression were used to identify variables associated with high PTE score, separately for adolescent men and women. Variables of interest with univariable $p$-values $<0.20$ were included in multivariable model selections. After testing for collinearity, only the sexual behaviour variable 'inconsistent condom use (yes vs. no vs. never had sex)' was considered for inclusion in the final model. For all other variables, model selections were performed using backward selection based on Type III $p$-values to reach the optimal (minimized) AIC. All statistical tests were considered statistically significant at $\alpha<0.05$.

\section{Results}

\section{Baseline characteristics}

Of 830 participants, 767 answered all 19 TESI-C items and were included in this analysis of whom 442 (58\%) were adolescent women and 325 (42\%) were adolescent men (Table 1). Median age was 17 years [Q1-Q3: 16-18], 45\% were Zulu, 85\% were currently enrolled in school, and 6\% had ever been incarcerated. A majority had lived in Soweto since birth (77\%), lived in brick house/flat owned by the family (71\%), reported high food insecurity (52\%), and lived in a household which had received a social grant in the last 12 months (57\%).

Overall, 56\% of participants reported having ever had sex, including $64 \%$ of adolescent men and $50 \%$ of adolescent women $(p<0.001$ for gender difference). Of those reporting sexual activity in the six months prior to the interview, 35\% reported having more than one sexual partner in the previous 6 months (including $56 \%$ of adolescent men and $18 \%$ of adolescent women $[p<0.001])$. Among those who had ever had sex, 54\% reported inconsistent condom use (including $53 \%$ of adolescent men and 55\% of adolescent women $[p=0.729])$ and $23 \%$ reported ever having been 
Table 1 Baseline characteristics of participants (aged 14-19 years) overall and by gender $(n=767)$

\begin{tabular}{|c|c|c|c|c|c|c|c|}
\hline \multirow[t]{2}{*}{ Baseline characteristics } & \multicolumn{2}{|c|}{ Overall $(n=767)$} & \multicolumn{2}{|c|}{ Adolescent Men $(n=325)$} & \multicolumn{2}{|c|}{ Adolescent Women $(n=442)$} & \multirow[t]{2}{*}{$p$-value } \\
\hline & $n$ & $\%$ & $n$ & $\%$ & $n$ & $\%$ & \\
\hline \multicolumn{8}{|l|}{ Socio-demographic characteristics } \\
\hline Age at interview (years, median, Q1,Q3) & 17 & 16,18 & 17 & 16,18 & 18 & 16,18 & 0.197 \\
\hline \multicolumn{8}{|l|}{ Years lived in Soweto } \\
\hline$<5$ years & 71 & 9.4 & 27 & 8.4 & 44 & 10.0 & \multirow[t]{4}{*}{0.347} \\
\hline$\geq 5$ years & 106 & 14.0 & 51 & 15.9 & 55 & 12.5 & \\
\hline Since birth & 582 & 76.7 & 242 & 75.6 & 340 & 77.5 & \\
\hline missing & 8 & & 5 & & 3 & & \\
\hline \multicolumn{8}{|l|}{ Ethnicity } \\
\hline Zulu & 345 & 45.0 & 166 & 51.1 & 179 & 40.5 & \multirow[t]{5}{*}{0.005} \\
\hline Xhosa & 92 & 12.0 & 39 & 12.0 & 53 & 12.0 & \\
\hline Sotho & 124 & 16.2 & 40 & 12.3 & 84 & 19.0 & \\
\hline Tswana & 85 & 11.1 & 26 & 8.0 & 59 & 13.4 & \\
\hline Other ethnicities & 121 & 15.8 & 54 & 16.6 & 67 & 15.2 & \\
\hline \multicolumn{8}{|l|}{ Education } \\
\hline$\geq$ High school & 9 & 1.2 & 7 & 2.2 & 2 & 0.5 & \multirow[t]{2}{*}{0.041} \\
\hline$<$ High school & 758 & 98.8 & 318 & 97.9 & 440 & 99.6 & \\
\hline \multicolumn{8}{|l|}{ Employment } \\
\hline Student & 649 & 85.1 & 264 & 81.5 & 385 & 87.7 & \multirow[t]{4}{*}{0.056} \\
\hline Unemployed & 85 & 11.1 & 44 & 13.6 & 41 & 9.3 & \\
\hline Employed & 29 & 3.8 & 16 & 4.9 & 13 & 3.0 & \\
\hline Missing & $<5$ & & $<5$ & & $<5$ & & \\
\hline \multicolumn{8}{|l|}{ Housing } \\
\hline Brick house/Flat owned by family & 547 & 71.3 & 220 & 67.7 & 327 & 74.0 & \multirow[t]{3}{*}{0.160} \\
\hline Brick house/Flat rented by family/other & 18 & 2.3 & 9 & 2.8 & 9 & 2.0 & \\
\hline RDP house/Shack & 202 & 26.3 & 96 & 29.5 & 106 & 24.0 & \\
\hline \multicolumn{8}{|l|}{ Food Insecurity } \\
\hline Low & 169 & 22.0 & 59 & 18.2 & 110 & 24.9 & \multirow[t]{3}{*}{0.078} \\
\hline Medium & 203 & 26.5 & 88 & 27.1 & 115 & 26.0 & \\
\hline High & 395 & 51.5 & 178 & 54.8 & 217 & 49.1 & \\
\hline \multicolumn{8}{|l|}{ Household Social Grant in the last 12 months } \\
\hline No & 325 & 42.9 & 141 & 44.3 & 184 & 41.9 & \multirow[t]{3}{*}{0.506} \\
\hline Yes & 432 & 57.1 & 177 & 55.7 & 255 & 58.1 & \\
\hline missing & 10 & & 7 & & 3 & & \\
\hline \multicolumn{8}{|l|}{ Incarceration history } \\
\hline No & 646 & 93.8 & 258 & 91.2 & 388 & 95.6 & \multirow[t]{3}{*}{0.019} \\
\hline Yes & 43 & 6.2 & 25 & 8.8 & 18 & 4.4 & \\
\hline Missing & 78 & & 42 & & 36 & & \\
\hline \multicolumn{8}{|l|}{ Sexual behaviour and HIV variables } \\
\hline \multicolumn{8}{|l|}{ Ever had sex } \\
\hline No & 338 & 44.1 & 116 & 35.7 & 222 & 50.2 & $<.001$ \\
\hline Yes & 429 & 55.9 & 209 & 64.3 & 220 & 49.8 & \\
\hline
\end{tabular}


Table 1 Baseline characteristics of participants (aged 14-19 years) overall and by gender $(n=767$ ) (Continued)

Sexually Active in the past 6 months $(L 6 M)^{a}$

$\begin{array}{llll}\text { No } & 153 & 36.5 & 80 \\ \text { Yes } & 266 & 63.1 & 12 \\ \text { missing } & 10 & & 7\end{array}$

Number of partners (among those reporting sexual activity in $L 6 M)^{b}$

\begin{tabular}{|c|c|c|c|c|c|c|c|}
\hline 1 partner & 168 & 64.6 & 51 & 43.6 & 117 & 81.8 & $<.001$ \\
\hline$\geq 2$ partner & 92 & 35.4 & 66 & 56.4 & 26 & 18.2 & \\
\hline Missing & 6 & & & & & & \\
\hline \multicolumn{8}{|l|}{ Condom use ${ }^{a}$} \\
\hline Consistent condom use & 189 & 46.3 & 93 & 47.2 & 96 & 45.5 & 0.729 \\
\hline Inconsistent condom use & 219 & 53.7 & 104 & 52.8 & 115 & 54.5 & \\
\hline missing & 21 & & 12 & & 9 & & \\
\hline \multicolumn{8}{|l|}{ HIV testing history } \\
\hline No & 414 & 54.1 & 187 & 57.7 & 227 & 51.5 & 0.087 \\
\hline Yes & 351 & 45.9 & 137 & 42.3 & 214 & 48.5 & \\
\hline \multicolumn{8}{|l|}{ HIV status (self-report) } \\
\hline HIV-positive & 11 & 1.4 & 5 & 1.5 & 6 & 1.4 & 0.187 \\
\hline HIV-negative & 329 & 42.9 & 127 & 39.1 & 202 & 45.7 & \\
\hline Unknown/never tested & 427 & 55.7 & 193 & 59.4 & 234 & 52.9 & \\
\hline \multicolumn{8}{|l|}{ STI or STI symptomology ${ }^{a}$} \\
\hline No & 332 & 77.4 & 173 & 82.8 & 159 & 72.3 & $<.001$ \\
\hline Yes & 97 & 22.6 & 36 & 17.2 & 61 & 27.7 & \\
\hline \multicolumn{8}{|c|}{ Substance use and mental health variables } \\
\hline \multicolumn{8}{|c|}{ Alcohol use in the last 6 months (L6M) } \\
\hline No & 267 & 34.99 & 104 & 32.1 & 163 & 37.1 & 0.150 \\
\hline Yes & 496 & 65.01 & 220 & 67.9 & 276 & 62.9 & \\
\hline \multicolumn{8}{|c|}{ Drug use in L6M (excluding marijuana use) } \\
\hline No & 728 & 94.9 & 297 & 91.4 & 431 & 97.5 & $<.001$ \\
\hline Yes & 39 & 5.1 & 28 & 8.6 & 11 & 2.5 & \\
\hline \multicolumn{8}{|l|}{ Probable Depression } \\
\hline No & 510 & 66.5 & 229 & 70.5 & 281 & 63.6 & 0.046 \\
\hline Yes (CES-D score $\geq 24$ ) & 257 & 33.5 & 96 & 29.5 & 161 & 36.4 & \\
\hline
\end{tabular}

Note: $p$-values in bold are significant $(<.05)$

Abbreviations: CES-D center for epidemiologic studies- depression scale, RDP reconstruction and development programme, STI sexually transmitted infection, HIV human immunodeficiency virus

${ }^{a}$ Among those reporting sexual activity ever

${ }^{\mathrm{b}}$ Among those reporting sexual activity in the last 6 month

diagnosed with an STI or experienced STI symptoms (including 17\% of adolescent men and $28 \%$ of adolescent women $[p=0.009])$. Overall, $1.4 \%$ reported being HIV-positive $(1.5 \%$ of adolescent men and $1.4 \%$ of women, $p=0.19$ ).

In the six months prior to interview, nearly twothirds (65\%) reported alcohol use and 5\% reported using other drugs. One-third (34\%) had probable depression, with higher rates among adolescent women than men ( $36 \%$ vs. $30 \%, p=0.05)$.

\section{Experience of potentially traumatic events (PTEs)}

Nearly all participants (99.7\%) reported experiencing at least 1 PTE. Median number of PTEs experienced was 7 [Q1-Q3: 5-9], with no significant difference by gender $(p=0.19)$. Overall, $47 \%$ of adolescent men and $45 \%$ of adolescent women experienced a high PTE score $(\geq 7$ events $(p=0.603))$.

Table 2 shows the proportion of adolescents who reported experiencing each of the 19 PTE items included in the adapted TESI-C scale by gender. Nearly three- 
quarters $(74 \%)$ of adolescent men and women reported experiencing the death of a family member or someone close to them. Over two-thirds (68\%) had witnessed a close family member or friend deal with a serious illness or injury. Nearly half reported that their parents were separated or divorced (48\%) or that their family struggled with money (46\%). In general, adolescent men were more likely to have experienced or perpetuated violent forms of traumatic experiences (e.g. forcing someone to have sex with them [7\%], deliberately inflicting harm on another [51\%], witnessed an act of violence in the community [76\%]). Adolescent women were more likely to experience psychological and emotional experiences of potentially traumatic events (e.g. having a family member have [46\%] or die from [41\%] HIV/AIDS).

Overall, $14 \%$ of adolescent women and $11 \%$ of adolescent men reported experiencing forced sex $(p=0.153)$ while $1.4 \%$ and $7.4 \%$ reported ever forcing someone to have sex with them $(p<0.001)$.

\section{Correlates of high PTE scores}

In unadjusted models among adolescent men (see Table 3), high PTE score was associated with older age, living in Soweto for $<5$ years, self-reported Tswana ethnicity, high food insecurity, drug use in the past six months, sexual experience, and inconsistent condom use. In the adjusted model (see Table 3), adolescent men with high PTE scores had significantly higher adjusted odds of being older $(\mathrm{aOR}=1.40$ /year, $95 \% \mathrm{CI}=1.21$ $1.63)$; recently moving to Soweto ( $<5$ years) vs. living in Soweto 'since birth' $(\mathrm{aOR}=2.78,95 \% \mathrm{CI}=1.14-6.76)$; and high vs. low food insecurity $(\mathrm{aOR}=2.6395 \% \mathrm{CI}=$ 1.36-5.09).

In the unadjusted models among adolescent women (see Table 4), high PTE score was associated with, high food insecurity, incarceration history, received a household social grant in the last year, probable depression, sexual experience and inconsistent condom use. In the adjusted model (see Table 4), adolescent women with high PTE scores had significantly higher adjusted odds of high food insecurity $(\mathrm{aOR}=2.57,95 \% \mathrm{CI}=1.55-4.26)$; probable depression $(\mathrm{aOR}=2.00,95 \% \mathrm{CI}=1.31-3.03)$; and inconsistent condom use vs. no sexual experience $(\mathrm{aOR}=2.69,95 \% \mathrm{CI}=1.66-4.37)$.

Table 2 Prevalence of potentially trauma event (PTE) experiences among participants (14-19 years) overall and by gender ( $n=767$ )

\begin{tabular}{|c|c|c|c|c|c|c|c|}
\hline & \multicolumn{2}{|c|}{$\begin{array}{l}\text { Overall } \\
(n=767)\end{array}$} & \multicolumn{2}{|c|}{$\begin{array}{l}\text { Adolescent Men } \\
(n=325)\end{array}$} & \multicolumn{2}{|c|}{$\begin{array}{l}\text { Adolescent Women } \\
(n=442)\end{array}$} & \multirow[t]{2}{*}{$p$-value } \\
\hline & $n$ & $\%$ & $n$ & $\%$ & $n$ & $\%$ & \\
\hline Experienced at least one PTE & 765 & 99.7 & 325 & 100.0 & 440 & 99.6 & 0.511 \\
\hline High trauma score $(\geq 7)($ alpha $=0.63)$ & 348 & 45.4 & 151 & 46.5 & 197 & 44.6 & 0.603 \\
\hline Separated from mom (e.g. lived with another relative or in foster care) & 253 & 33.0 & 118 & 36.3 & 135 & 30.5 & 0.093 \\
\hline Parents separated & 370 & 48.2 & 153 & 47.1 & 217 & 49.1 & 0.581 \\
\hline Parents argued frequently or more than usual & 259 & 33.8 & 111 & 34.2 & 148 & 33.5 & 0.846 \\
\hline Changed schools (not because of graduation) or moved to a new home & 245 & 31.9 & 123 & 37.9 & 122 & 27.6 & 0.003 \\
\hline Parent/guardian lost job & 342 & 44.6 & 139 & 42.8 & 203 & 45.9 & 0.385 \\
\hline Lost home or had no home & 65 & 8.5 & 38 & 11.7 & 27 & 6.1 & 0.006 \\
\hline Family member or someone close had HIV/AIDS & 287 & 37.4 & 85 & 26.2 & 202 & 45.7 & $<0.001$ \\
\hline Family member or someone close died of HIV/AIDS & 273 & 35.6 & 91 & 28.0 & 182 & 41.2 & 0.001 \\
\hline Family member or someone close died & 569 & 74.2 & 243 & 74.8 & 326 & 73.8 & 0.751 \\
\hline Family member or someone close was very sick or had a bad injury & 524 & 68.3 & 230 & 70.8 & 294 & 66.5 & 0.211 \\
\hline Experienced race/ethnicity discrimination & 183 & 23.9 & 77 & 23.7 & 106 & 24.0 & 0.926 \\
\hline Family struggled with money & 355 & 46.3 & 147 & 45.2 & 208 & 47.1 & 0.616 \\
\hline Seen an act of violence towards someone else (not in family) & 538 & 70.1 & 248 & 76.3 & 290 & 65.6 & 0.001 \\
\hline Experienced an act of violence by someone not in your family & 316 & 41.2 & 147 & 45.2 & 169 & 38.2 & 0.052 \\
\hline Seen an act of violence in the family & 324 & 42.2 & 136 & 41.9 & 188 & 42.5 & 0.849 \\
\hline Experienced an act of violence by someone in your family & 240 & 31.3 & 107 & 32.9 & 133 & 30.1 & 0.403 \\
\hline Deliberately inflicted harm on another person & 293 & 38.2 & 166 & 51.1 & 127 & 28.7 & $<0.001$ \\
\hline Experienced forced Sex & 98 & 12.8 & 35 & 10.8 & 63 & 14.3 & 0.153 \\
\hline Experienced forcing someone to have sex & 30 & 3.9 & 24 & 7.4 & 6 & 1.4 & $<0.001$ \\
\hline
\end{tabular}


Table 3 Univariate and adjusted analysis of variables associated with high PTE scores among adolescent men $(n=325)$

\begin{tabular}{|c|c|c|c|c|c|c|c|c|c|c|c|}
\hline \multirow[b]{2}{*}{ Variables } & \multicolumn{2}{|c|}{$\begin{array}{l}\text { Low PTE } \\
\text { score }\end{array}$} & \multicolumn{2}{|c|}{$\begin{array}{l}\text { High PTE } \\
\text { score }\end{array}$} & \multirow{2}{*}{$\begin{array}{l}p \text {-value } \\
\text { Wilcoxon/Chisq }\end{array}$} & \multicolumn{6}{|c|}{ High PTE score vs. Low PTE score } \\
\hline & $\bar{n}$ & $\%$ & $\bar{n}$ & $\%$ & & $\overline{\mathrm{OR}}$ & $95 \% \mathrm{C}$ & & $\mathrm{AOR}$ & $95 \% \mathrm{C}$ & \\
\hline \multicolumn{12}{|l|}{ Socio-demographic characteristic } \\
\hline Age at interview (per year, median Q1,Q3) & 17 & 15,18 & 18 & 16,18 & $<.001$ & 1.37 & 1.19 & 1.59 & 1.40 & 1.21 & 1.63 \\
\hline \multicolumn{12}{|l|}{ Years lived in Soweto } \\
\hline Since birth & 133 & 76.9 & 109 & 74.2 & 0.059 & Ref & & & Ref & & \\
\hline$\geq 5$ years & 31 & 17.9 & 20 & 13.6 & & 0.79 & 0.42 & 1.46 & 0.75 & 0.39 & 1.43 \\
\hline$<5$ years & 9 & 5.2 & 18 & 12.2 & & 2.44 & 1.05 & 5.65 & 2.78 & 1.14 & 6.76 \\
\hline \multicolumn{12}{|l|}{ Ethnicity } \\
\hline Zulu & 99 & 56.9 & 67 & 44.4 & 0.174 & Ref & & & & & \\
\hline Xhosa & 18 & 10.3 & 21 & 13.9 & & 1.72 & 0.85 & 3.48 & Not Selected & & \\
\hline Sotho & 21 & 12.1 & 19 & 12.6 & & 1.34 & 0.67 & 2.67 & & & \\
\hline Tswana & 10 & 5.8 & 16 & 10.6 & & 2.36 & 1.01 & 5.52 & & & \\
\hline Other ethnicities & 26 & 14.9 & 28 & 18.5 & & 1.59 & 0.86 & 2.95 & & & \\
\hline \multicolumn{12}{|l|}{ Employment } \\
\hline Student & 147 & 85.0 & 117 & 77.5 & 0.193 & Ref & & & & & \\
\hline Unemployed & 20 & 11.56 & 24 & 15.89 & & 1.51 & 0.79 & 2.86 & Not Selected & & \\
\hline Employed & 6 & 3.5 & 10 & 6.6 & & 2.09 & 0.74 & 5.93 & & & \\
\hline \multicolumn{12}{|l|}{ Housing } \\
\hline Brick house/Flat owned by family & 123 & 70.7 & 97 & 64.2 & 0.414 & Ref & & & & & \\
\hline Brick house/Flat rented by family/Hostel/Other & 5 & 2.9 & 4 & 2.7 & & 1.01 & 0.27 & 3.88 & & & \\
\hline RDP house/Shack & 46 & 26.44 & 50 & 33.11 & & 1.38 & 0.85 & 2.23 & & & \\
\hline \multicolumn{12}{|l|}{ Food Insecurity } \\
\hline Low & 39 & 22.4 & 20 & 13.3 & 0.026 & Ref & & & Ref & & \\
\hline Medium & 51 & 29.3 & 37 & 24.5 & & 1.41 & 0.71 & 2.81 & 1.58 & 0.76 & 3.29 \\
\hline High & 84 & 48.3 & 94 & 62.3 & & 2.18 & 1.18 & 4.03 & 2.63 & 1.36 & 5.09 \\
\hline \multicolumn{12}{|l|}{ Household Social Grant } \\
\hline No & 81 & 47.9 & 60 & 40.3 & 0.170 & Ref & & & Not Selected & & \\
\hline Yes & 88 & 52.1 & 89 & 59.7 & & 1.37 & 0.87 & 2.13 & & & \\
\hline \multicolumn{12}{|l|}{ Incarceration history } \\
\hline No & 148 & 92.5 & 110 & 89.4 & 0.367 & Ref & & & & & \\
\hline Yes & 12 & 7.5 & 13 & 10.6 & & 1.46 & 0.64 & 3.32 & & & \\
\hline \multicolumn{12}{|l|}{ Sexual behaviour and HIV } \\
\hline \multicolumn{12}{|l|}{ HIV testing history } \\
\hline No & 99 & 57.2 & 88 & 58.3 & 0.848 & Ref & & & & & \\
\hline Yes & 74 & 42.8 & 63 & 41.7 & & 0.96 & 0.62 & 1.49 & & & \\
\hline \multicolumn{12}{|l|}{ HIV Result } \\
\hline Positive & 3 & 1.7 & 2 & 1.3 & 0.940 & Ref & & & & & \\
\hline Negative & 69 & 39.7 & 58 & 38.4 & & 1.26 & 0.20 & 7.81 & & & \\
\hline Unknown/Never tested & 102 & 58.6 & 91 & 60.3 & & 1.34 & 0.22 & 8.19 & & & \\
\hline \multicolumn{12}{|l|}{ Sex Ever } \\
\hline No & 77 & 44.3 & 39 & 25.8 & 0.001 & Ref & & & Not included ${ }^{a}$ & & \\
\hline Yes & 97 & 55.8 & 112 & 74.2 & & 2.28 & 1.42 & 3.65 & & & \\
\hline
\end{tabular}


Table 3 Univariate and adjusted analysis of variables associated with high PTE scores among adolescent men $(n=325)$ (Continued)

\begin{tabular}{|c|c|c|c|c|c|c|c|c|c|}
\hline \multicolumn{10}{|l|}{ Ever STI } \\
\hline No & 85 & 48.9 & 88 & 58.3 & 0.001 & Ref & & & Not included ${ }^{\mathrm{a}}$ \\
\hline Yes & 12 & 6.9 & 24 & 15.9 & & 1.93 & 0.91 & 4.11 & \\
\hline Never had sex & 77 & 44.3 & 39 & 25.8 & & 0.49 & 0.30 & 0.80 & \\
\hline \multicolumn{10}{|l|}{ Sexually Active P6M } \\
\hline No & 41 & 24.1 & 39 & 26.4 & 0.001 & Ref & & & Not Included ${ }^{a}$ \\
\hline Yes & 52 & 30.6 & 70 & 47.3 & & 1.42 & 0.80 & 2.49 & \\
\hline Never had sex & 77 & 45.3 & 39 & 26.4 & & 0.53 & 0.30 & 0.95 & \\
\hline \multicolumn{10}{|l|}{ Inconsistent condom use } \\
\hline Never had sex & 77 & 45.8 & 39 & 26.9 & 0.002 & Ref & & & Not Selected \\
\hline No & 44 & 26.2 & 49 & 33.8 & & 2.20 & 1.26 & 3.85 & \\
\hline Yes & 47 & 28.0 & 57 & 39.3 & & 2.39 & 1.39 & 4.13 & \\
\hline \multicolumn{10}{|l|}{ More than 1 partner in the L6M } \\
\hline No & 23 & 13.6 & 28 & 19.4 & 0.016 & Ref & & & Not Included ${ }^{\mathrm{a}}$ \\
\hline Yes & 28 & 16.6 & 38 & 26.4 & & 1.11 & 0.53 & 2.33 & \\
\hline Never had sex/Sexually inactive & 118 & 69.8 & 78 & 54.2 & & 0.54 & 0.29 & 1.01 & \\
\hline \multicolumn{10}{|c|}{ Substance use and mental health variables } \\
\hline \multicolumn{10}{|l|}{ Alcohol use in L6M } \\
\hline No & 63 & 36.4 & 41 & 27.2 & 0.075 & 1.09 & 0.47 & 2.52 & \\
\hline Yes & 110 & 63.6 & 110 & 72.9 & & 0.65 & 0.28 & 1.51 & \\
\hline \multicolumn{10}{|l|}{ Probable Depression } \\
\hline No & 129 & 74.1 & 100 & 66.2 & 0.119 & Ref & & & Not Selected \\
\hline Yes (score $\geq 24$ ) & 45 & 25.9 & 51 & 33.8 & & 1.54 & 0.96 & 2.47 & \\
\hline \multicolumn{10}{|c|}{ Drug use ever in L6M (excluding marijuana use) } \\
\hline No & 165 & 94.8 & 132 & 87.4 & 0.018 & Ref & & & Not Selected \\
\hline Yes & 9 & 5.2 & 19 & 12.6 & & 2.64 & 1.16 & 6.02 & \\
\hline
\end{tabular}

Note: AORs and $p$-values in bold are significant $(<.05)$

Abbreviations: $C l$ confidence intervals, OR odds ratio, $A O R$ adjusted odds ratio, CES-D center for epidemiologic studies- depression scale, $R D P$ reconstruction and development programme, STI, sexually transmitted infection, HIV human immunodeficiency virus

${ }^{a}$ Not included due to Collinearity

\section{Discussion}

Similar to other South African and African studies $[8,45]$, we found that adolescents in our study experienced high levels of PTEs. Nearly all participants experienced at least one PTE (99.7\%) and had experienced on average 7 PTEs at the time of their interview with no differences by gender. A study of U.S adolescents (aged 13-17) found that $61.8 \%$ had lifetime PTE experience [46], compared with $99.7 \%$ of adolescents within our study. Among both adolescent men and women, increased exposure to PTE was associated with high levels of food insecurity. This finding has implications for sexual and reproductive health (SRH) outcomes and overall well-being for South African adolescent men and women faced with syndemic risks including high levels of community-level violence and sexual victimization [21].
In addition, our findings suggest no difference in the prevalence of PTEs between adolescent men and women, rather differences in the types of traumatic occurrences. Despite no significant differences in PTE prevalence by gender, we pursued a gender stratified analysis to enable examination of differential correlates of experiencing multiple PTEs. These findings highlight a need for future research to explore the differential potential gendered impacts of PTEs experienced among adolescents.

Consistent with previous literature, we found that PTE exposure and the effects are distributed along social and gendered axes. For example, a number of studies globally have found that young women are more likely to experience sexual assault while men are more likely to experience physical assault $[29,31,45]$. 
Table 4 Univariate and adjusted analysis of variables associated with high PTE scores among adolescent women ( $n=442)$

\begin{tabular}{|c|c|c|c|c|c|c|c|c|c|c|c|}
\hline \multirow[b]{2}{*}{ Variables } & \multicolumn{2}{|c|}{ Low PTE score } & \multicolumn{2}{|c|}{ High PTE score } & \multirow{2}{*}{$\begin{array}{l}p \text {-value } \\
\text { Wilcoxon/Chisq }\end{array}$} & \multicolumn{6}{|c|}{ High PTE score vs. Low PTE score } \\
\hline & $n$ & $\%$ & $n$ & $\%$ & & $\overline{\mathrm{OR}}$ & $95 \% \mathrm{Cl}$ & & $\mathrm{AOR}$ & $95 \% C$ & \\
\hline \multicolumn{12}{|l|}{ Socio-demographic characteristics } \\
\hline Age & 17 & 16,18 & 18 & 16,18 & 0.182 & 1.10 & 0.97 & 1.24 & Not Selected & & \\
\hline \multicolumn{12}{|l|}{ Years lived in Soweto } \\
\hline$<5$ years & 22 & 9.0 & 22 & 11.3 & 0.511 & Ref & & & & & \\
\hline$\geq 5$ years & 28 & 11.5 & 27 & 13.9 & & 0.96 & 0.44 & 2.13 & & & \\
\hline Since birth & 194 & 79.5 & 146 & 74.9 & & 0.75 & 0.40 & 1.41 & & & \\
\hline \multicolumn{12}{|l|}{ Ethnicity } \\
\hline Zulu & 104 & 42.5 & 75 & 38.1 & 0.764 & Ref & & & & & \\
\hline Xhosa & 29 & 11.8 & 24 & 12.2 & & 1.15 & 0.62 & 2.13 & & & \\
\hline Sotho & 48 & 19.6 & 36 & 18.3 & & 1.04 & 0.62 & 1.76 & & & \\
\hline Tswana & 29 & 11.8 & 30 & 15.2 & & 1.43 & 0.79 & 2.59 & & & \\
\hline Other & 35 & 14.3 & 32 & 16.2 & & 1.27 & 0.72 & 2.23 & & & \\
\hline \multicolumn{12}{|l|}{ Employment } \\
\hline Student & 217 & 89.7 & 168 & 85.3 & 0.379 & Ref & & & & & \\
\hline Unemployed & 19 & 7.85 & 22 & 11.17 & & 1.50 & 0.78 & 2.85 & & & \\
\hline Employed & 6 & 2.5 & 7 & 3.6 & & 1.51 & 0.50 & 4.57 & & & \\
\hline \multicolumn{12}{|l|}{ Housing } \\
\hline House owned by family & 184 & 75.1 & 143 & 72.6 & 0.577 & Ref & & & & & \\
\hline House rented by family/Other & 6 & 2.5 & 3 & 1.5 & & 0.64 & 0.16 & 2.62 & & & \\
\hline RDP house/Shack & 55 & 22.45 & 51 & 25.89 & & 1.19 & 0.77 & 1.85 & & & \\
\hline \multicolumn{12}{|l|}{ Food Insecurity } \\
\hline Low & 77 & 31.4 & 33 & 16.8 & $<.001$ & Ref & & & Ref & & \\
\hline Medium & 71 & 29.0 & 44 & 22.3 & & 1.45 & 0.83 & 2.52 & 1.49 & 0.84 & 2.65 \\
\hline High & 97 & 39.6 & 120 & 60.9 & & 2.89 & 1.77 & 4.70 & 2.57 & 1.55 & 4.26 \\
\hline \multicolumn{12}{|c|}{ Household ever Received Social Grant } \\
\hline No & 112 & 46.1 & 72 & 36.7 & 0.048 & Ref & & & Not Selected & & \\
\hline Yes & 131 & 53.9 & 124 & 63.3 & & 1.47 & 1.00 & 2.16 & & & \\
\hline \multicolumn{12}{|l|}{ Sexual behaviour and HIV variables } \\
\hline \multicolumn{12}{|l|}{ HIV testing history } \\
\hline No & 139 & 57.0 & 88 & 44.7 & 0.010 & Ref & & & Not Selected & & \\
\hline
\end{tabular}

Sexual behaviour and HIV variables

HIV testing history

$\begin{array}{llll}139 & 57.0 & 88 & 44.7 \\ 105 & 43.0 & 109 & 55.3\end{array}$

0.010

Ref

Not Selected

Yes

$1.64 \quad 1.12$

2.39

HIV Result

$\begin{array}{lllll}\text { Positive } & 3 & 1.2 & 3 & 1.5 \\ \text { Negative } & 102 & 41.6 & 100 & 50.8 \\ & 140 & 57.1 & 94 & 47.7\end{array}$

0.131 Ref

Unknown

$\begin{array}{llll}140 & 57.1 & 94 & 47.7\end{array}$

$\begin{array}{lll}0.98 & 0.19 & 4.97\end{array}$

$\begin{array}{lll}0.67 & 0.13 \quad 3.40\end{array}$

Sex Ever

$\begin{array}{lllllllll}\text { No } & 142 & 58.0 & 80 & 40.6 & <.001 & \text { Ref } & \\ \text { Yes } & 103 & 42.0 & 117 & 59.4 & & 2.02 & 1.38 & 2.95 \\ \text { TI or STI symptomology } & & & & & & & & \\ \text { No } & 80 & 32.7 & 79 & 40.1 & <.001 & \text { Ref } & \\ \text { Yes } & 23 & 9.4 & 38 & 19.3 & & 1.67 & 0.91 & 3.06 \\ \text { Never had sex } & 142 & 58.0 & 80 & 40.6 & & 0.57 & 0.38 & 0.86\end{array}$


Table 4 Univariate and adjusted analysis of variables associated with high PTE scores among adolescent women $(n=442)$ (Continued)

\begin{tabular}{|c|c|c|c|c|c|c|c|c|c|c|c|}
\hline \multicolumn{12}{|l|}{ Sexually Active L6M } \\
\hline No & 41 & 16.9 & 32 & 16.3 & $<.001$ & Ref & & & & & \\
\hline Yes & 60 & 24.7 & 84 & 42.9 & & 1.79 & 1.02 & 3.17 & & & \\
\hline Never had sex & 142 & 58.4 & 80 & 40.8 & & 0.72 & 0.42 & 1.24 & & & \\
\hline \multicolumn{12}{|l|}{ Inconsistent condom use } \\
\hline Never had sex & 142 & 59.7 & 80 & 41.0 & $<.001$ & Ref & & & Ref & & \\
\hline No & 52 & 21.9 & 44 & 22.6 & & 1.50 & 0.92 & 2.44 & 1.59 & 0.96 & 2.63 \\
\hline Yes & 44 & 18.5 & 71 & 36.4 & & 2.86 & 1.80 & 4.56 & 2.69 & 1.66 & 4.37 \\
\hline \multicolumn{12}{|l|}{ More than 1 partner in L6M } \\
\hline No & 49 & 20.2 & 68 & 34.9 & $<.001$ & Ref & & & Not included* & & \\
\hline Yes & 11 & 4.5 & 15 & 7.7 & & 0.98 & 0.42 & 2.32 & & & \\
\hline Never had sex/Sexually inactive & 183 & 75.3 & 112 & 57.4 & & 0.44 & 0.29 & 0.68 & & & \\
\hline \multicolumn{12}{|c|}{ Substance use and mental health variables } \\
\hline \multicolumn{12}{|l|}{ Alcohol Use in the L6M } \\
\hline No & 102 & 42.0 & 61 & 31.1 & 0.019 & Ref & & & Not Selected & & \\
\hline Yes & 141 & 58.0 & 135 & 68.9 & & 1.60 & 1.08 & 2.38 & & & \\
\hline \multicolumn{12}{|l|}{ Probable Depression } \\
\hline No & 176 & 71.8 & 105 & 53.3 & $<.001$ & Ref & & & Ref & & \\
\hline Yes (score $\geq 24$ ) & 69 & 28.2 & 92 & 46.7 & & 2.23 & 1.51 & 3.32 & 2.00 & 1.31 & 3.03 \\
\hline \multicolumn{12}{|l|}{ Incarceration history } \\
\hline No & 226 & 97.4 & 162 & 93.1 & 0.037 & Ref & & & Not Selected & & \\
\hline Yes & 6 & 2.6 & 12 & 6.9 & & 2.79 & 1.03 & 7.59 & & & \\
\hline \multicolumn{12}{|c|}{ Drug use ever in L6M (excluding marijuana use) } \\
\hline No & 239 & 97.6 & 192 & 97.5 & 0.952 & Ref & & & & & \\
\hline Yes & 6 & 2.5 & 5 & 2.5 & & 1.04 & 0.31 & 3.45 & & & \\
\hline
\end{tabular}

Note: AORs in bold are significant $(<.05)$

Abbreviations: $C l$ confidence intervals; $O R$ odds ratio, $A O R$ adjusted odds ratio, CES-D center for epidemiologic studies- depression scale, $R D P$ reconstruction and development programme, STI sexually transmitted infection, HIV human immunodeficiency virus

*Not included due to Collinearity

\section{Adolescent women}

Our results align with previous research indicating that co-occuring multiple PTEs experienced by women influence heightened depression symptomology [8], and compound syndemic risks of HIV transmission through increased HIV risk behaviour such as inconsistent condom use $[10,30]$. The synergistic effect of multiple experiences of PTEs and increased HIV acquisition risk may be exacerbated among women living in vulnerable urban environments, such as Soweto, facing economic hardships and high levels of food insecurity [23, 30]. These compounding experiences of structural vulnerability influence economic dependence - placing women in inferior roles in their relationships - in turn increasing experiences of gender-based violence, inability to negotiate condom use, and ultimately HIV transmission risk $[3,23]$.

\section{Adolescent men}

Our results indicate that high-PTE scores were more commonly found among older adolescent men who have recently moved to Soweto, and who face high levels of food insecurity. Experiences of trauma can accumulate over the lifecourse, [47], as such older age was a hypothesized finding for higher number of PTEs among men in our study. The exposure to multiple experiences of PTEs at a young age have been found to perpetuate aggressive behaviour and negative views towards women in adulthood $[48,49]$. The development of negative views towards women may perpetuate harmful gender norms and inequitable power dynamics in relationships, which has shown to have significant implications for the HIV epidemic in South Africa [24, 50-52]. Furthermore, young men living in South Africa face extremely high rates of interpersonal violence. A study assessing hospital data on 
injuries within the Mthatha Hospital Complex in South Africa, found that the majority of injuries occurred among men, with $60 \%$ of all cases being for acts of interpersonal violence [27]. Despite extremely high levels of PTEs within men participating in our study, we found that this was not significantly associated with increased depression symptomology or inconsistent condom use. Previous research has explored the relationship between high levels of trauma and post-traumatic growth [53]. Resilience to HIV risk among adolescent men living in HIV hyper-endemic nations experiencing concurrent poverty and high-levels of PTEs should be further explored.

\section{Intervention implications}

Reducing syndemic risks to traumatic experiences in both adolescent men and women is likely to have a positive impact on HIV transmission through multiple pathways. The scale-up of community and structural level interventions, as well as increased focus on trauma-informed models of care for adolescents in South Africa is critical for addressing the HIV epidemic [21, 54]. For adolescent women, intervention strategies aimed at increasing economic independence, reducing gender-based violence, reducing inequities in relationship power and control, and challenging gender norms, are critical to increase sustained and widespread uptake of HIV prevention options, including male and female condoms and, in more recent years, pre-exposure prophylaxis (PrEP), [48, 55-57]. Among adolescent women, high rates of sexual violence and inequities in relationship power [50, 58, 59] intersect to compromise opportunities to negotiate condom use [30, 60-62]. Given demonstrated links between trauma, poor mental health, and sexual behaviours, mediated through pathways of gender and power inequity, central to the efforts to reduce HIV incidence among adolescent women is a clear need to scale-up access to youthcentred, trauma-informed, and women-controlled HIV prevention strategies, inclusive of PrEP [4].

Trauma-focused cognitive behavioural therapy (TFCBT) has been shown to be highly beneficial in reducing sexual health risk. Hien and colleagues [63] implemented a skill-based TF-CBT program focusing on various domains including: personal self-management, coping, communication, boundary setting, HIV risk reduction and reducing unsafe behaviour in general. Women in the trauma-focused intervention were almost half as likely to report unprotected sex compared to women in the control group [63]. Given the high number of PTEs experienced by young people in South Africa, it is imperative to scale-up such trauma-informed mental health services for adolescents [21].

Community-level interventions addressing harmful gender norms, such as Stepping Stones, have been successful at reducing the perpetuation of intimate partner violence, a significant step forward in reducing HIV transmission and experiences of trauma for adolescent women [48]. For both adolescent men and women, interventions aimed at addressing food insecurities may help to mediate the compounding affects of PTEs on HIV transmission within vulnerable urban environments such as Soweto. This relationship merits further examination. Future interventions should consider the importance of resilience and post-traumatic growth within settings where experiences of traumatic events and HIV risk are extremely high [64].

\section{Strengths \& limitations}

In conducting a gender-stratified analysis of PTE occurrence, we demonstrated the multitude of implications that PTEs have on both SRH programs and HIV intervention - informing a gendered approach to addressing PTE and HIV risk. However, we did not include measurements within our survey to assess PTSD symptomology which is a known outcome of experiencing trauma $[8,10,21]$, thus we acknowledge this is a limitation of our study which should be further examined within future South African adolescent health studies. Further, we are unable to assess causation within this cross-sectional study. Additional limitations include recall and social desirability bias due to self-reported measures of sexual behaviour and other sensitive topics. In addition, we used a modified variation of the TESI$\mathrm{C}$; therefore, caution should be used in comparing these findings with other studies using the original version of the TESI-C and other scales similarly measuring experiences of trauma.

\section{Conclusion}

Being an adolescent in Soweto, South Africa poses many challenges: we found a high prevalence of PTEs along with associations highlighting risk for HIV acquisition, particularly for adolescent women. Adolescence is a dynamic and transitional time of the lifecourse, marked by rapid and multiple developmental changes that, through biology and socialization, are distinctly gendered $[5,65,66]$. Enabling and fostering the pathway towards health provides adolescent men and women with a set of meaningful skills and coping mechanisms that they can carry into adulthood [5, 21]. Focusing on preventing multiple co-occurring risks and promoting increased access to mental health services for adolescent men and women facing high exposures to PTEs can begin to address the syndemic of HIV and trauma which pose significant threats to HIV-acquisition, population health and development for South Africa [10]. 


\section{Additional file}

Additional file 1: Comparison of potentially traumatic event items assessed within the Botsha Bophelo Adolescent Health Survey and TESI-C items. (DOCX $101 \mathrm{~kb}$ )

\begin{abstract}
Abbreviations
AIC: Akaike information criterion; AIDS: Acquired immune deficiency syndrome; aOR: Adjusted odds ratio; ART: Antiretroviral therapy; BBAHS: Botsha Bophelo adolescent health survey; CES-D: Centre for epidemiologic studies depression; HIV: Human immunodeficiency virus; KMAC: Kganya Motsha Adolescent Centre; PHRU: Perinatal HIV research unit; PLHIV: People living with HIV/AIDS; PrEP: Pre-exposure prophylaxis; PTEs: Potentially traumatic events; PTSD: Post-traumatic stress disorder; STIs: Sexually transmitted infections; TasP: Treatment as prevention; TESIC: Traumatic event screening inventory-child (TESI-C); TF-CBT: Traumafocused cognitive behavioural therapy
\end{abstract}

\section{Acknowledgement}

The Botsha Bophelo Adolescent Health Study (BBAHS) Research Team would like to thank our participants and our research team members for all their contributions to this study.

\section{Funding}

BBAHS was funded by the Canadian Institutes of Health Research (CIHR), Institute for Human Development, Child and Youth Health (230513). Initial seed funding was provided by Simon Fraser University through a President Research Award to CLM. NJL is supported by a CANFAR/CTN Postdoctoral Fellowship Award. AK received salary support from the Canada Research Chair program in Global Perspectives on HIV and Sexual and Reproductive Health. The PHRU was supported through a grant by the South African Medical Research Council. The authors have no conflict of interest to declare regarding the publication of this manuscript.

\section{Availability of data and materials}

For access to the study data, please contact Dr. Cari Miller (Cari.Miller@sfu.ca), Principal Investigator of the Botsha Bophelo Adolescent Health Study.

\section{Authors' contributions}

CLM, JD, BN, GG, RSH and AK designed the study. JD, BN, and GG implemented the study. RSH and CLM had full access to the data in the study and take responsibility for the integrity of the data and the accuracy of the data analysis. JC undertook the data analysis and ZC conducted the statistical analysis. KC, AM and AK interpreted the data and wrote the first draft of the manuscript. All authors read and approved the final manuscript.

\section{Competing interests}

The authors declare that they have no competing interests.

\section{Consent for publication}

This manuscript does not contain any identifying individual participant data, and thus consent for publication from participants in not applicable for this analysis.

\section{Ethics approval and consent to participate}

Ethical approval for the study was granted by the ethics committees of the University of the Witwatersrand (Johannesburg, South Africa) [M090449] and Simon Fraser University (Burnaby, Canada) [\#2009 s0196]. Adolescents under 18 years signed an informed assent form and provided a signed informed consent form from a parent or legal guardian. Adolescents aged 18 or 19 signed an informed consent form.

\section{Author details}

${ }^{1}$ Faculty of Health Sciences, Simon Fraser University (SFU), Blusson Hall Rm 10522, 8888 University Drive, Burnaby BC V5A 1S6, Canada. ${ }^{2}$ British Columbia Centre for Excellence in HIV/AIDS, Vancouver, Canada. ${ }^{3}$ Perinatal HIV Research Unit (PHRU), Faculty of Health Sciences, University of the Witwatersrand, Johannesburg, South Africa. ${ }^{4}$ Faculty of Humanities, University of the Witwatersrand, Johannesburg, South Africa. ${ }^{5}$ School of Public Health and Social Policy, University of Victoria, Victoria, Canada.
Received: 21 May 2016 Accepted: 12 November 2016 Published online: 25 November 2016

\section{References}

1. UNICEF. United Nations Children's Fund. Statistics| South Africa. 2013. http:// www.unicef.org/infobycountry/southafrica_statistics.html.

2. UNAIDS. Global AIDS response progress report 2012: Republic of South Africa. 2012

3. Dellar RC, Dlamini S, Karim QA. Adolescent girls and young women: key populations for HIV epidemic control. J Int AIDS Soc. 2015;18(2 Suppl 1):19408.

4. Venter WD, Cowan F, Black V, Rebe K, Bekker LG. Pre-exposure prophylaxis in Southern Africa: feasible or not? J Int AIDS Soc. 2015;18(4 Suppl 3):19979.

5. Bekker LG, Johnson L, Wallace M, Hosek S. Building our youth for the future J Int AIDS Soc. 2015;18(Suppl 1):20027 http://www.jiasociety.org/index.php/ jias/article/view/20027 |http://dx.doi.org/10.7448/IAS.18.2.20027.

6. Meinck F, Cluver LD, Boyes ME, Mhlongo EL. Risk and protective factors for physical and sexual abuse of children and adolescents in Africa: a review and implications for practice. Trauma Violence Abuse. 2015;16(1):81-107.

7. Pence BW, Shirey K, Whetten K, Agala B, Itemba D, Adams J, Whetten R, Yao J, Shao J. Prevalence of psychological trauma and association with current health and functioning in a sample of HIV-infected and HIV-uninfected Tanzanian adults. PLoS One. 2012:7(5), e36304.

8. Suliman S, Mkabile SG, Fincham DS, Ahmed R, Stein DJ, Seedat S. Cumulative effect of multiple trauma on symptoms of posttraumatic stress disorder, anxiety, and depression in adolescents. Compr Psychiatry. 2009;50(2):121-7.

9. Whetten K, Shirey K, Pence BW, Yao J, Thielman N, Whetten R, Adams J, Agala B, Ostermann J, O'Donnell K, et al. Trauma history and depression predict incomplete adherence to antiretroviral therapies in a low income country. PLoS One. 2013;8(10), e74771.

10. Brezing C, Ferrara M, Freudenreich O. The syndemic illness of HIV and trauma: implications for a trauma-informed model of care. Psychosomatics. 2015;56(2):107-18.

11. Neller DJ, Denney RL, Pietz CA, Thmlinson RP. Testing the Trauma Model of Violence. J Fam Viol. 2005:3

12. Khan MR, Kaufman JS, Pence BW, Gaynes BN, Adimora AA, Weir SS, Miller WC. Depression, Sexually Transmitted Infection, and Sexual Risk Behavior Among Young Adults in the United States. Arch Pediatr Adolesc Med. 2009;163(7):644.

13. Brawner BM, Gomes MM, Jemmott LS, Deatrick JA, Coleman CL. Clinical depression and HIV risk-related sexual behaviors among AfricanAmerican adolescent females: unmasking the numbers. AIDS Care. 2012; 24(5):618-25.

14. Shrier LA, Harris SK, Sternberg M, Beardslee WR. Associations of depression, self-esteem, and substance use with sexual risk among adolescents. Prev Med. 2001;33(3):179-89.

15. Hutton HE, Lyketsos CG, Zenilman JM, Thompson RE, Erbelding EJ. Depression and HIV risk behaviors among patients in a sexually transmitted disease clinic. Am J Psychiatry. 2004;161(5):912-4.

16. Gibbs A, Willan S, Sikwyiya Y, Jama Shai N, Washington L. Experiences of childhood trauma increases HIV-risk behaviours in young women and men in urban informal settlements in South Africa. Durban: World AIDS Conference 2016; 2016.

17. Nduna M, Jewkes RK, Dunkle KL, Shai NP, Colman I. Associations between depressive symptoms, sexual behaviour and relationship characteristics: a prospective cohort study of young women and men in the Eastern Cape, South Africa. J Int AIDS Soc. 2010;13:44.

18. Barhafumwa B, Dietrich J, Closson K, Samji H, Cescon A, Nkala B, Davis J, Hogg RS, Kaida A, Gray G, et al. High prevalence of depression symptomology among adolescents in Soweto, South Africa assocaited with being female and cofactors relating to HIV transmission. Vulnerable Child Youth Stud. 2016;11(3):263-73.

19. Millstein B. Introduction to the Syndemics Prevention Network 2002. Accessed 14th Nov 2016. [http://www2.cdc.gov/syndemics/PPT/ An\%20Introduction\%20to\%20Syndemics\%20(as\%20delivered).ppt].

20. Batchelder AW, Gonzalez JS, Palma A, Schoenbaum E, Lounsbury DW. A Social Ecological Model of Syndemic Risk affecting Women with and At-Risk for HIV in Impoverished Urban Communities. Am J Community Psychol. 2015:56(3-4):229-40

21. Kaminer D, Hardy A, Heath K, Mosdell J, Bawa U. Gender patterns in the contribution of different types of violence to posttraumatic stress symptoms among South African urban youth. Child Abuse Negl. 2013;37(5):320-30. 
22. Abrahams N, Devries K, Watts C, Pallitto C, Petzold M, Shamu S, GarcíaMoreno C. Worldwide prevalence of non-partner sexual violence: a systematic review. Lancet. 2014;383(9929):1648-54.

23. Decker MR, Peitzmeier S, Olumide A, Acharya R, Ojengbede O, Covarrubias L, Gao E, Cheng Y, Delany-Moretlwe S, Brahmbhatt H. Prevalence and Health Impact of Intimate Partner Violence and Non-partner Sexual Violence Among Female Adolescents Aged 15-19 Years in Vulnerable Urban Environments: A Multi-Country Study. J Adolesc Health. 2014;55(6 Suppl):S58-67.

24. Jewkes R, Sikweyiya Y, Morrell R, Dunkle K. Gender inequitable masculinity and sexual entitlement in rape perpetration South Africa: findings of a cross-sectional study. PLoS One. 2011;6(12):e29590.

25. Jewkes $\mathrm{R}$, Abrahams $\mathrm{N}$. The epidemiology of rape and sexual coercion in South Africa: an overview. Soc Sci Med. 2002;55:1231-44.

26. Dunkle KL, Jewkes RK, Brown HC, Yoshihama M, Gray GE, McIntyre JA, Harlow SD. Prevalence and patterns of gender-based violence and revictimization among women attending antenatal clinics in Soweto, South Africa. Am J Epidemiol. 2004;160(3):230-9.

27. Dhaffala A, Longo-Mbenza B, Kingu JH, Peden M, Kafuko-Bwoye A, Clarke M, Mazwai EL. Demographic profile and epidemiology of injury in Mthatha, South Africa. Afr Health Sci. 2013;13(4):1144-8.

28. Otwombe KN, Dietrich J, Sikkema KJ, Coetzee J, Hopkins KL, Laher F, Gray GE. Exposure to and experiences of violence among adolescents in lower socioeconomic groups in Johannesburg, South Africa. BMC Public Health. 2015;15:450.

29. Norris FH. Epidemiology of trauma: frequency and impact of different potentially traumatic events on different demographic groups. J Consult Clin Psychol. 1992;60(3):409-18.

30. Pitpitan EV, Kalichman SC, Eaton LA, Cain D, Sikkema KJ, Watt MH, Skinner D, Pieterse D. Co-occurring psychosocial problems and HIV risk among women attending drinking venues in a South African township: a syndemic approach. Ann Behav Med. 2013;45(2):153-62.

31. Amir M, Sol O. Psychological impact and prevalence of traumatic events in a student sample in Israel: the effect of multiple traumatic events and physical injury. J Trauma Stress. 1999;12(1):139-54.

32. Census 2011- main place "Soweto" [https://www.citypopulation.de/php/ southafrica-cityofjohannesburg.php?cid=798026].

33. Nkala B, Khunwane M, Dietrich J, Otwombe K, Sekoane I, Sonqishe B, Gray G. Kganya Motsha Adolescent Centre: a model for adolescent friendly HIV management and reproductive health for adolescents in Soweto, South Africa. AIDS Care. 2015;27(6):697-702.

34. Dietrich J, Khunwane M, Laher F, de Bruyn G, Sikkema KJ, Gray G. "Group sex" parties and other risk patterns: A qualitative study about the perceptions of sexual behaviors and attitudes of adolescents in Soweto, South Africa. Vulnerable Child Youth Stud. 2011;6(3):244-54

35. Dietrich J, Sikkema K, Otwombe KN, Sanchez A, Nkala B, de Bruyn G, Van Der Watt M, Gray GE. Multiple levels of influence in predicting sexual activity and condom use among adolescents in Soweto, Johannesburg, South Africa. J HIV AIDS Soc Serv. 2013;12(3-4):404-23.

36. Otwombe KN, Sikkema KJ, Dietrich J, de Bruyn G, van der Watt M, Gray GE. Willingness to participate in biomedical HIV prevention studies after the HVTN 503/Phambili trial: a survey conducted among adolescents in Soweto, South Africa. J Acquir Immune Defic Syndr. 2011;58(2):211-8.

37. Miller CL, Nkala B, Closson K, Chia J, Cui Z, Palmer A, Hogg RS, Gray G, Dietrich J. The Botsha Bophelo Adolescent Health Study (BBAHS): a profile of a South African - Canadian research collaboration. PLoS One. 2016. In Press

38. Gray MJ, Slagle DM. Selecting a Potentially Traumatic Event Screening Measure. J Trauma Pract. 2006:5(2):1-19.

39. Kendall A, Olson CM, Edward A, Frongillo J. Validation of the Radimer/ Cornell Measures of HUnger and Food Security. J Nutr. 1995;125:2793-801.

40. Radloff LS. The CES-D scale a self-report depression scale for research in the general population. Appl Psychol Meas. 1977;1(3):385-401.

41. Lewinsohn PM, Seeley JR, Roberts RE, Allen NB. Center for Epidemiologic Studies Depression Scale (CES-D) as a screening instrument for depression among community-residing older adults. Psychol Aging. 1997;12(2):277-87.

42. Chabrol H, Montovany A, Chouicha K, Duconge E. Study of the CES-D on a sample of 1,953 adolescent students. L'Encéphale. 2002;28(5 Pt 1):429-32.

43. Bamidele JO, Asekun-Olarinmoye EO, Odu OO, Amusan OA, Egbewale BE. Sociodemographic characteristics and health risk behaviours among students of a tertiary institution in south western Nigeria. Afr J Med Med Sci. 2007;36(2):129-36.
44. van Heerden MS, Grimsrud AT, Seedat S, Myer L, Williams DR, Stein DJ. Patterns of substance use in South Africa: results from the South African Stress and Health study. S Afr Med J. 2009;99(5 Pt 2):358-66.

45. Peltzer K. Predictors of positive health among a sample of South African adolescents. Psychol Rep. 2007;100(3 Pt 2):1186-8.

46. McLaughlin KA, Koenen KC, Hill ED, Petukhova M, Sampson NA, Zaslavsky AM, Kessler RC. Trauma exposure and posttraumatic stress disorder in a national sample of adolescents. J Am Acad Child Adolesc Psychiatry. 2013; 52(8):815-30. e814.

47. Global Burden of Disease Pediatrics C, Kyu HH, Pinho C, Wagner JA, Brown JC, Bertozzi-Villa A, Charlson FJ, Coffeng LE, Dandona L, Erskine HE, et al. Global and National Burden of Diseases and Injuries Among Children and Adolescents Between 1990 and 2013: Findings From the Global Burden of Disease 2013 Study. JAMA Pediatrics. 2016.

48. Jewkes R, Nduna M, Levin J, Jama N, Dunkle K, Puren A, Duvvury N. Impact of stepping stones on incidence of HIV and HSV-2 and sexual behaviour in rural South Africa: cluster randomised controlled trial. BMJ. 2008;337:a506.

49. Hinsberger M, Sommer J, Kaminer D, Holtzhausen L, Weierstall R, Seedat S, Madikane S, Elbert T. Perpetuating the cycle of violence in South African low-income communities: attraction to violence in young men exposed to continuous threat. Eur J psychotraumatol. 2016;7:29099.

50. Jewkes R, Nduna M, Jama-Shai N, Chirwa E, Dunkle K. Understanding the Relationships between Gender Inequitable Behaviours, Childhood Trauma and Socio-Economic Status in Single and Multiple Perpetrator Rape in Rural South Africa: Structural Equation Modelling. PLoS One. 2016;11(5):e0154903.

51. Jewkes RK, Dunkle K, Nduna M, Shai N. Intimate partner violence, relationship power inequity, and incidence of HIV infection in young women in South Africa: a cohort study. Lancet. 2010;376:41-8.

52. Jewkes R, Morrell R. Gender and sexuality: emerging perspectives from the heterosexual epidemic in South Africa and implications for HIV risk and prevention. J Int AIDS Soc. 2010;13:6.

53. Sherr L, Nagra N, Kulubya G, Catalan J, Clucas C, Harding R. HIV infection associated post-traumatic stress disorder and post-traumatic growth-a systematic review. Psychol Health Med. 2011;16(5):612-29.

54. Murray LK, Skavenski S, Kane JC, Mayeya J, Dorsey S, Cohen JA, Michalopoulos LT, Imasiku M, Bolton PA. Effectiveness of Trauma-Focused Cognitive Behavioral Therapy Among Trauma-Affected Children in Lusaka, Zambia: A Randomized Clinical Trial. JAMA Pediatr. 2015;169(8):761-9.

55. Pronyk PM, Hargreaves JR, Kim JC, Morison LA, Phetla G, Watts C, Busza J, Porter JDH. Effect of a structural intervention for the prevention of intimatepartner violence and HIV in rural South Africa: a cluster randomised trial. Lancet. 2006:368(9551):1973-83.

56. Harrison A, Colvin CJ, Kuo C, Swartz A, Lurie M. Sustained High HIV Incidence in Young Women in Southern Africa: Social, Behavioral, and Structural Factors and Emerging Intervention Approaches. Curr HIV/AIDS Rep. 2015;12(2):207-15.

57. Pettifor A, Nguyen NL, Celum C, Cowan FM, Go V, Hightow-Weidman L. Tailored combination prevention packages and PrEP for young key populations. J Int AIDS Soc. 2015;18(2 Suppl 1):19434.

58. Jewkes RK, Levin JB, Penn-Kekana LA. Gender inequalities, intimate partner violence and HIV preventive practices: findings of a South African crosssectional study. Soc Sci Med. 2003;56(1):125-34.

59. Petersen I, Bhana A, McKay M. Sexual violence and youth in South Africa: the need for community-based prevention interventions. Child Abuse Negl. 2005;29(11):1233-48

60. Schuyler AC, Masvawure TB, Smit JA, Beksinska M, Mabude Z, Ngoloyi C, Mantell JE. Building young women's knowledge and skills in female condom use: lessons learned from a South African intervention. Health Educ Res. 2016;31(2):260-72.

61. Bhana A, Zimmerman R, Cupp P. Gender role attitudes and sexual risk among adolescents in South Africa. Vulnerable Child Youth Stud. 2008;3:112-9.

62. Swan $\mathrm{H}, \mathrm{O}^{\prime} \mathrm{Connell} \mathrm{DJ}$. The impact of intimate partner violence on women's condom negotiation efficacy. J Interpers Violence. 2012;27(4):775-92.

63. Hien DA, Campbell AN, Killeen T, Hu MC, Hansen C, Jiang H, Hatch-Maillette M, Miele GM, Cohen LR, Gan W, et al. The impact of trauma-focused group therapy upon HIV sexual risk behaviors in the NIDA Clinical Trials Network "Women and trauma" multi-site study. AIDS Behav. 2010;14(2):421-30.

64. van der Walt L, Suliman S, Martin L, Lammers K, Seedat S. Resilience and post-traumatic stress disorder in the acute aftermath of rape: a comparative 
analysis of adolescents versus adults. J Child Adolesc Mental health. 2014;26(3):239-49.

65. Igras SM, Macieira M, Murphy E, Lundgren R. Investing in very young adolescents' sexual and reproductive health. Glob Public Health. 2014;9(5):555-69.

66. Johansson EE, Alex L, Christianson M. Gendered discourses of youth sexualities-an exploration of PubMed articles on prevention of sexually transmissible infections. Sex Reprod Healthc. 2014;5(3):81-9.

Submit your next manuscript to BioMed Central and we will help you at every step:

- We accept pre-submission inquiries

- Our selector tool helps you to find the most relevant journal

- We provide round the clock customer support

- Convenient online submission

- Thorough peer review

- Inclusion in PubMed and all major indexing services

- Maximum visibility for your research

Submit your manuscript at www.biomedcentral.com/submit 